\title{
Author Correction: Lineage-specific RUNX2 super-enhancer activates MYC and promotes the development of blastic plasmacytoid dendritic cell neoplasm
}

\author{
Sho Kubota (1) 13, Kenji Tokunaga 2,13, Tomohiro Umezu (1) ${ }^{3}$, Takako Yokomizo-Nakano ${ }^{1}$, Yuqi Sun', \\ Motohiko Oshima ${ }^{4,5}$, Kar Tong Tan ${ }^{6}$, Henry Yang ${ }^{6}$, Akinori Kanai ${ }^{7}$, Eisaku Iwanaga ${ }^{2}$, Norio Asou $^{8}$, \\ Takahiro Maeda9 ${ }^{9}$ Naomi Nakagata ${ }^{10}$, Atsushi Iwama ${ }^{4,5}$, Kazuma Ohyashiki ${ }^{3}$, Motomi Osato6,11,12 \& \\ Goro Sashida (1) ${ }^{1}$
}

Correction to: Nature Communications https://doi.org/10.1038/s41467-019-09710-z, published online 10 April 2019.

The original version of this Article contained an error in the spelling of the author Kar Tong Tan, which was incorrectly given as Kar Ton Tan. This has now been corrected in both the PDF and HTML versions of the Article.

Published online: 28 August 2019

\begin{abstract}
(i) Open Access This article is licensed under a Creative Commons Attribution 4.0 International License, which permits use, sharing, adaptation, distribution and reproduction in any medium or format, as long as you give appropriate credit to the original author(s) and the source, provide a link to the Creative Commons license, and indicate if changes were made. The images or other third party material in this article are included in the article's Creative Commons license, unless indicated otherwise in a credit line to the material. If material is not included in the article's Creative Commons license and your intended use is not permitted by statutory regulation or exceeds the permitted use, you will need to obtain permission directly from the copyright holder. To view a copy of this license, visit http://creativecommons.org/licenses/by/4.0/.
\end{abstract}

(C) The Author(s) 2019

\footnotetext{
${ }^{1}$ Laboratory of Transcriptional Regulation in Leukemogenesis, International Research Center for Medical Sciences (IRCMS), Kumamoto University, 2-2-1 Honjo, Chuo Ward, Kumamoto 860-0811, Japan. ${ }^{2}$ Department of Hematology, Kumamoto University, 1-1-1 Honjo, Chuo Ward, Kumamoto 860-8556, Japan. ${ }^{3}$ Department of Hematology, Tokyo Medical University, 6-7-1 Nishi-Shinjuku, Shinjuku, Tokyo 160-0023, Japan. ${ }^{4}$ Department of Cellular and Molecular Medicine, Chiba University, 1-8-1 Inohana, Chuo Ward, Chiba 260-8670, Japan. ${ }^{5}$ Division of Stem Cell and Molecular Medicine, Center for Stem Cell Biology and Regenerative Medicine, The Institute of Medical Science, The University of Tokyo, 4-6-1 Shirokanedai, Minato, Tokyo 108-8639, Japan. ${ }^{6}$ Cancer Science Institute of Singapore, National University of Singapore, Singapore 119077, Singapore. ${ }^{7}$ Department of Molecular Oncology, Research Institute for Radiation Biology and Medicine, Hiroshima University, Hiroshima 739-0046, Japan. ${ }^{8}$ Department of Hematology, International Medical Center, Saitama Medical University, Saitama 350-1298, Japan. ${ }^{9}$ Department of General Medicine, Nagasaki University, Graduate School of Biomedical Science, Nagasaki 852-8523, Japan. ${ }^{10}$ Division of Reproductive Engineering, Center for Animal Resources and Development (CARD), Kumamoto University, 2-2-1 Honjo, Chuo Ward, Kumamoto 860-0811, Japan. ${ }^{11}$ Laboratory of Runx Biology, International Research Center for Medical Sciences (IRCMS), Kumamoto University, 2-2-1 Honjo, Chuo Ward, Kumamoto 860-0811, Japan. ${ }^{12}$ Center for Metabolic Regulation of Healthy Aging (CMHA), Kumamoto University, Chuo Ward, Kumamoto 860-0811, Japan. ${ }^{13}$ These authors contributed equally: Sho Kubota, Kenji Tokunaga. Correspondence and requests for materials should be addressed to M.O. (email: csimo@nus.edu.sg) or to G.S. (email: sashidag@kumamoto-u.ac.jp)
} 